\title{
A Run-to-Run Control Framework for VLSI Manufacturing
}

\author{
Nauman Chaudhry, Roland Telfeyan, Brian Moore, Hossein Etemad and James Moyne \\ Center for Integrated Sensors and Circuits \\ Electrical Engineering and Computer Science Department \\ The University of Michigan \\ Ann Arbor, Michigan 48109-2122 \\ 313-936-3645 moyne@eecs.umich.edu
}

\begin{abstract}
A framework has been developed for the Run-to-Run (R2R) control and optimization of VLSI manufacturing processes. The framework is one component of the Michigan Sequential Control and OPtimization Environment (M-SCOPE). MSCOPE in-turn is part of a multi-level control system that includes real-time equipment and process control as well as pseudo-real-time process control components, operating in conjunction with the sequential control component. A feasibility implementation of the R2R framework has been developed for the optimization and control of a plasma etching process in a Reactive Ion Etcher. Results indicate process control robustness and improved process control versus open loop operation.
\end{abstract}

\section{Introduction}

The issue of R2R or sequential optimization and control of (VLSI manufacturing) processes has been receiving considerable attention over the past few years. The emphasis in the VLSI applications arena has been on the development and implementation of algorithms for discrete process control based on statistical process control (SPC) theory $[1,2]$. These algorithms however represent only one form of technology available for use in a single aspect of $\mathrm{R} 2 \mathrm{R}$ process optimization and control. A number of other methods and corresponding software solutions are available for implementation in discrete control systems [3]. Examples include neural networks, expert systems, and fuzzy logic controllers $[1,2,4]$. The ranges of use of each of these methods (including SPC), though generally overlapping, do vary greatly. Thus, in an ideal discrete control system, a number of these methods could be utilized in a complementary fashion.

The above control technologies are generally mature with feasibility implementations developed $[1,2,3]$. Their widespread adoption into the industry however has been slow; this process is being hindered by a lack of a generic run-time framework that allows for the incorporation of these control algorithm implementations along with other elements of sequential control. It is clear then that the resolution of a framework design is critical to the development of a portable and transferable R2R control system.

In this paper, a generic framework design for VLSI process and equipment $\mathrm{R} 2 \mathrm{R}$ control is described that provides an environment for the utilization of any number of discrete control mechanisms in a complementary fashion. The general control environment (in which this framework resides) is also described. A discussion is included of a typical framework implementation for R2R control; this description includes an overview of the various components that would in general be incorporated into the design, and a listing of the advantages of this generic system over traditional implementations. Results are presented on a feasibility implementation of the R2R framework that illustrate the advantages of the control system over open loop operation. This paper concludes with a summary and a discussion of future efforts in this area.

\section{Design Environment}

A R2R control framework design described in this paper represents one component in a much larger sequential control environment referred to as M-SCOPE (Michigan Control and OPtimization Environment) [3]. M-SCOPE in-turn is part of a multi-level control system that includes real-time equipment and process control as well as pseudo-real-time process control components operating in conjunction with the sequential control component. A typical implementation of this multi-level control system is illustrated in Figure 1. Note that the process control problem in this case is broken down into three distinct components: in-situ process factory control, in-situ process control, and discrete product control. In the ideal case these three control loops operate in a hierarchical feedback fashion as shown. Unfortunately, research into in-situ control in VLSI manufacturing is in its infancy and should not be considered yet as a transferable technology [3]. Thus state-of-the art generic control systems in this arena must be capable of providing R2R control without in-situ control. Eventually, however, as in-situ control reaches maturity, the generic control system should provide a migration path for the incorporation of this additional control capability (see Figure 1).

A review of current trends in control and automation reveals many other design requirements of the R2R framework. The framework should be (as much as possible) generic and should accommodate all requirements of the R2R control system. Further, the design should be compatible with current trends in control, standardization, and VLSI manufacturing. Finally, and perhaps most importantly, any solution should have the capability to easily incorporate existing components that could be utilized in a R2R control system (e.g., commercial 
statistical advice packages, communication drivers and process monitors, graphical user interface packages, etc.). Specifically it should be capable of utilizing any number of control and optimization methodologies simultaneously in a complementary fashion.

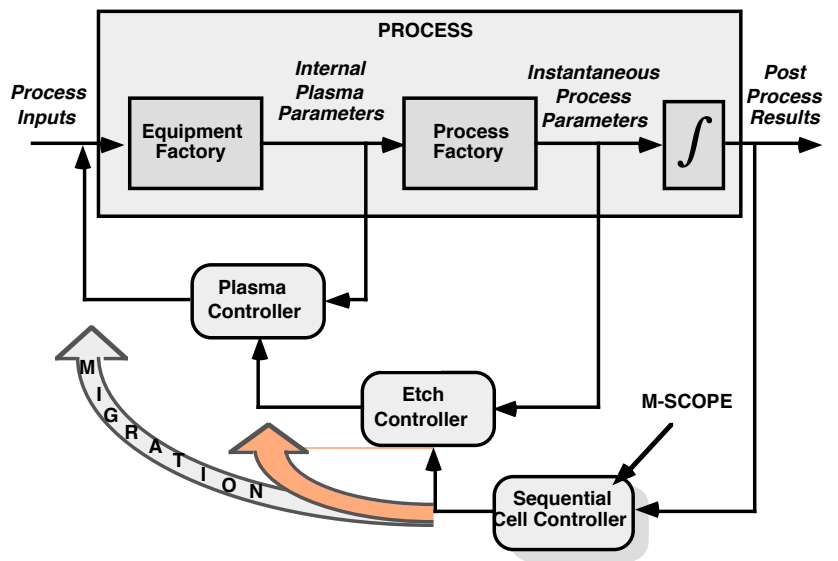

Figure 1: Multi-level Hierarchical Control System

\section{Controller Design}

A framework design conforming to the above specifications has been developed at The University of Michigan and is illustrated in Figure 2 [3]. The design features a Generic Cell Controller (GCC) mechanism at its core [5]. This mechanism utilizes a relational database to store the sequential control algorithm(s). For this reason the GCC is capable of supporting complex control algorithms that are characteristic of many R2R control systems. Further, due to GCC database interaction specifications, a very high degree of modularity is established with GCC applications. This results in both high portability and transferability of software, and a capability to easily and arbitrarily incorporate commercially available R2R control components into the system. Finally, it is important to note that the GCC framework is truly generic as it is hardware, software, communications, and implementation independent; and the framework supports current trends in control and VLSI manufacturing such as SEMI Generic Equipment Models (GEMs) [6].

The GCC mechanism serves as the "glue" to coordinate the information flow between process modules so as to achieve run-to-run control. (These modules include process monitoring and diagnostics, metrology, communications, and process optimization and control.) The R2R control algorithm itself is illustrated in Figure 3. Note that the algorithm is multi-thread, i.e., any number of available control and optimization packages may be utilized in a complementary fashion in any one implementation.

Referring to Figures 2 and 3, the R2R control loop can be traced as follows. Following the completion of the "n-th" process, metrology data is collected on process target parameters. These parameters are then compared with the actual targets. Using this information along with pertinent historical information (from previous runs), determinations are made as to the state of the process. These determinations include: (1), whether the process is in need of further optimization, (2), the regime of control or optimization through which the process is being navigated, and (3) the class of (available) optimization and control mechanisms would be best suited to modify the process recipe. The appropriate optimization - control mechanism(s) are then invoked and recipe advices obtained. These advices are convolved in a weighted fashion and composite recipe advice is obtained. The appropriate optimization and control models are then updated and the new recipe advice is available for download to the equipment for the " $n+1$ " process.

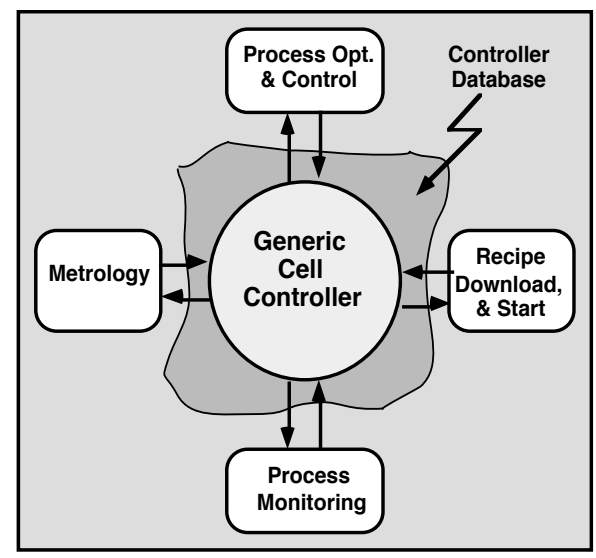

Figure 2: Run-to-Run Control Framework

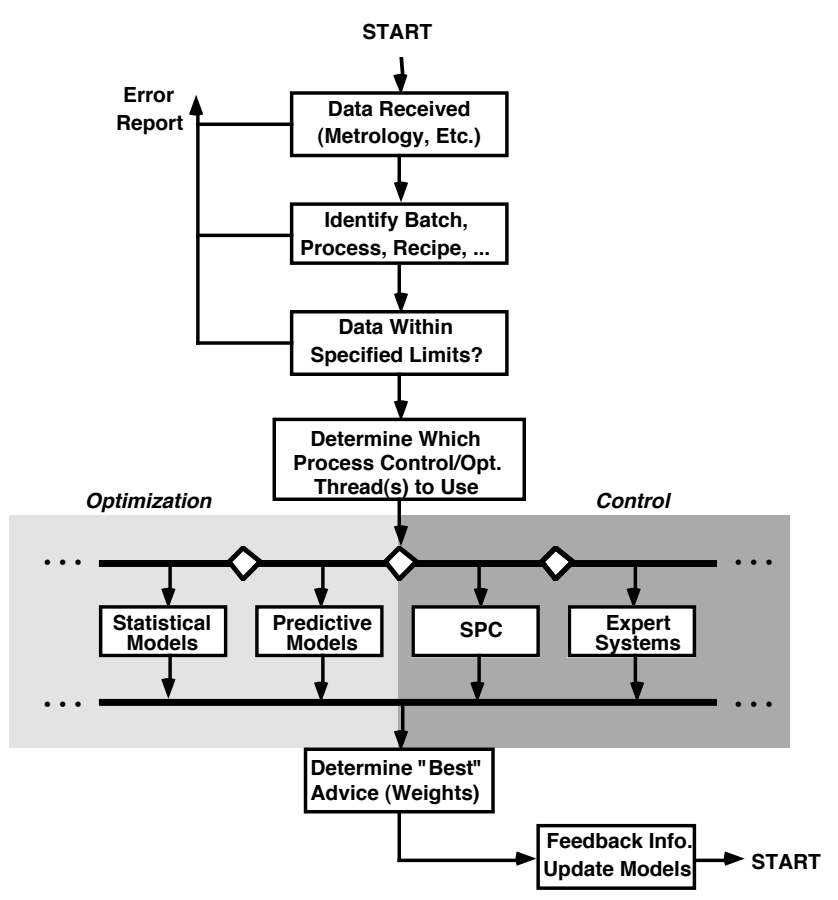

Figure 3: Run-to-Run Control Algorithm

\section{Implementation Results}

A feasibility implementation of the R2R framework has been developed for the optimization / control of a plasma etching 
process in an Applied Materials 8300 Reactive Ion Etcher. The optimization / control scheme incorporates three threads: (1), a statistical optimization / model building commercial package called ULTRAMAX, (2), a optimization / model building package that utilizes in-situ process data, and (3), a first order SPC algorithm developed at MIT. Experiments were conducted in which the R2R controller was utilized for the optimization and control of the measured parameters of etch rate and over etch depth, through the setting and modification of the input (recipe) parameters of etch time and oxygen flow. Other etch recipe parameters such as power, pressure, and various gas flows were kept constant. Results indicate that the R2R framework implementation does achieve $\mathrm{R} 2 \mathrm{R}$ process optimization and control through the utilization of all three optimization / control threads. Specifically, when no in-situ data is available the ULTRAMAX thread is invoked to search for a process optimum. However when this in-situ data is available, the in-situ optimization thread is utilized to develop process models and move towards a process target optimum. First order approximations are then used by the SPC thread to maintain that optimum. Experimental results, partially illustrated in Figure 4, clearly indicate both an improvement over open loop operation, and robustness in the face of sensor drift as well as process target shifts.
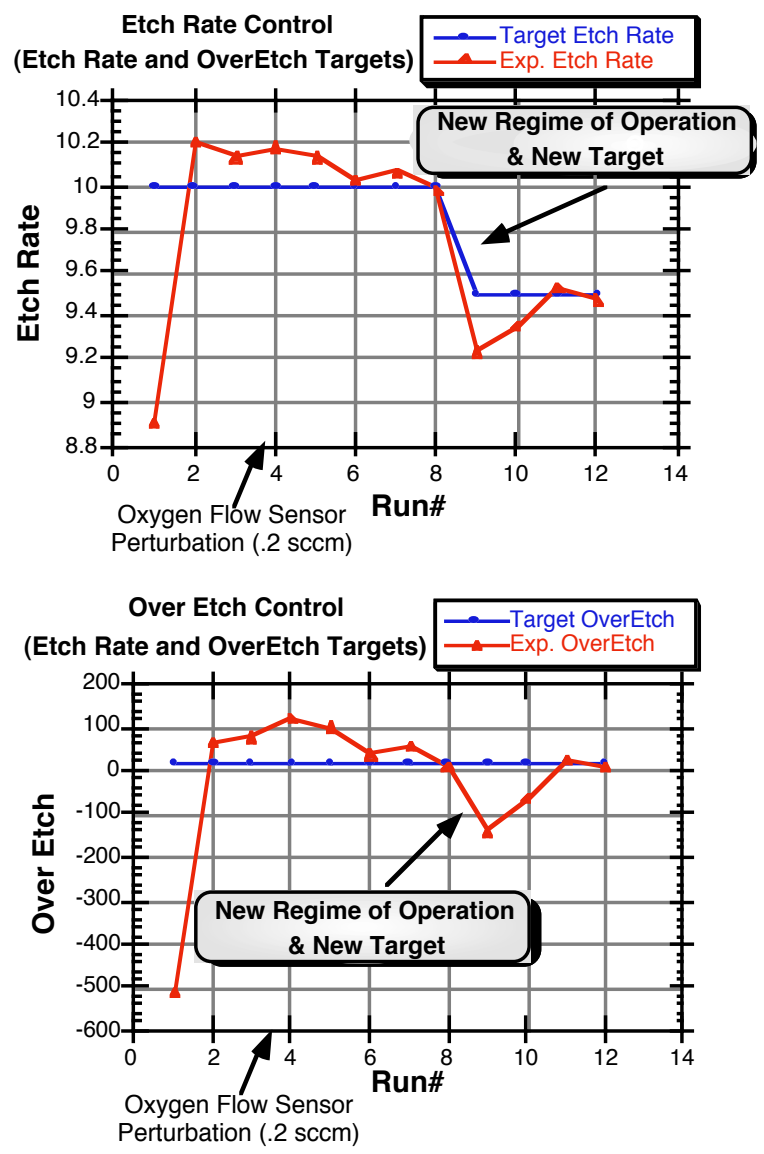

Figure 4: Experimental Results in Process Control

\section{Conclusions}

A framework has been conceptualized, designed and developed for the R2R control and optimization of VLSI manufacturing processes. The resulting design is very generic and modular; attractive features include ability to easily incorporate commercial control software and communication packages, software and hardware independence, a high degree of portability and modularity, and a capability to handle complex control algorithms using a relational database. The R2R control algorithm incorporated into the framework is multithread, and thus allows the complementary utilization of multiple control and optimization methodologies in a single implementation. Results of a feasibility implementation of the framework, developed for the optimization / control of a plasma etching process, indicate significant improvement over open loop operation.

In the future, research in this area will continue in the development of control and optimization threads as well as algorithms that can provide for the complementary utilization of these threads in a R2R system. Research will also continue into the further development of the GCC core to support fuzzy control as well as to support and enhance in-situ control. In the technology transfer area, work is continuing on the development of a complete M-SCOPE R2R software system that could be transferred easily to industry.

\section{Acknowledgments}

The authors gratefully acknowledge the contributions to this effort of Sureka Satyam, Jeff Fournier, Brian Rashap, Keith Bowerman, Mike Elta and Fred Terry. This research is supported by the Semiconductor Research Corporation and ARPA.

\section{References}

[1] E. Sachs and A. Hu, "Run by run process control: Combining SPC and Feedback Control," (submitted to) IEEE Transactions on Semiconductor Manufacturing, (Submitted October 1991).

[2] C.J. Spanos, "Statistical process control in semiconductor manufacturing," Proceedings of IEEE, (June 1992).

[3] J. Moyne, "A run-to-run control framework for VLSI manufacturing: A white paper," Technical Report \#213, Center For Integrated Sensors and Circuits, Department of Electrical Engineering and Computer Science, The University of Michigan, Ann Arbor, (February 1993).

[4] Lee, C.C., "Fuzzy logic in control systems: Fuzzy Logic Controller -- Part 1, Part 2," IEEE Transactions on Systems, Man, and Cybernetics, (March 1990).

[5] J.R. Moyne and L.C. McAfee, Jr. "A generic cell controller for the automated VLSI manufacturing facility," IEEE Transactions on Semiconductor Manufacturing, (May 1992).

[6] E30-92, The GEM Standard, 1992 SEMI Standards, Semiconductor Equipment and Materials International., (September 1992). 